\title{
Impacto de la inclusión de artículos escritos en inglés en revistas biomédicas españolas de edición multilingüe
}

\section{Impact of English-written articles included in multilingual-edited Spanish journals}

\author{
R. Cremades Pallas ${ }^{1}$, P. Burbano ${ }^{2}$, M.A. Valcárcel de La Iglesia ${ }^{1}$, G. Burillo-Putze ${ }^{3}$, \\ F.J. Martín-Sánchez ${ }^{1}$, Ò. Mirón ${ }^{2,4}$
}

\section{RESUMEN}

Objetivos. Analizar el impacto que tienen los artículos publicados en inglés, en comparación con los publicados en castellano, por revistas biomédicas españolas de edición multilingüe.

Metodología. De los artículos publicados entre 20082012 en 5 revistas españolas biomédicas multilingües, se analizó el idioma, el número de originales, la nacionalidad de la autoría, las citas recibidas, los artículos citantes y la nacionalidad de los autores citantes.

Resultados. Se incluyeron 4.296 documentos, 85 de ellos publicados en inglés (2\%). Estos últimos tuvieron unos porcentajes significativamente superiores de originales y de artículos sin autoría española, y consiguieron más citas y más artículos citantes por artículo publicado.

Conclusiones. La proporción de artículos publicados en inglés por las revistas españolas multilingües es bajo, con mayor frecuencia son originales y firmados exclusivamente por autores extranjeros, y reciben más citas que los publicados en español, que son realizadas también más frecuentemente por autores extranjeros.

Palabras clave. Investigación. Publicación. Factor de impacto. Lengua española. Lengua inglesa.

\begin{abstract}
Objectives. To analyze the impact which articles published in English have compared to those published in Spanish in multilingual Spanish biomedical journals.

Methodology. We analyzed the language of publication, the number of original articles, the nationality of the authors, the citations received, the citing article and the nationality of the citing authors among the articles published from 2008-2012 in 5 multilingual Spanish biomedical journals.
\end{abstract}

Results. The study included 4,296 documents, 85 of which were published in English (2\%). The percentage of original articles and of non Spanish authorship was significantly higher among these latter articles and they also achieved more citations and more citing articles per article published.

Conclusions. The proportion of articles published in English by multilingual Spanish biomedical journals is low and they are more often originals signed exclusively by foreign authors and receive more citations than those published in Spanish, which are also more frequently done by foreign authors.

Key words. Investigation. Publication. Impact factor. Spanish language. English language.
1. Servicio de Urgencias. Hospital Clínico San Carlos. Madrid.

2. Grupo de Investigación "Urgencias: procesos y patologías". IDIBAPS. Barcelona.

3. Servicio de Urgencias. Hospital Universitario de Canarias. Tenerife.

4. Área de Urgencias. Hospital Clínic. Barcelona.

\section{Correspondencia:}

Òscar Miró

Área de Urgencias

Hospital Clínic

Villarroel 170

08036 Barcelona

omiro@clinic.cat 


\section{INTRODUCCIÓN}

Actualmente, se estima que el número revistas científicas de publicación periódica en el mundo sobrepasa las 100.000, de las cuales poco más de 10.000 están indexadas en los principales repertorios bibliométricos. Dentro de este repertorio se encuentra la base Science Citation Index Expanded (SCI-Expanded), que en 2010 recogía 2.582 revistas biomédicas ${ }^{1}$. De ellas, $2256(87,4 \%)$ se editan en exclusivamente en inglés. Este porcentaje es tan elevado porque no solo las editan revistas en los países de habla inglesa, sino que muchas de países no anglosajones optan también por editarse en inglés ante las ventajas que conlleva: mayor posibilidad de recibir manuscritos de autores anglosajones (con mayor tradición y liderazgo en investigación) y mayor penetración entre sus lectores (con mayores posibilidades de expandir su horizonte a nuevos potenciales lectores más allá de sus fronteras). La situación en España puede resumirse en tres posibilidades: revistas que se editan completamente en inglés, revistas que se editan completamente en español y revistas multilingües. Estas últimas publican simultáneamente artículos en español y en inglés, en función del idioma en el que los autores envían su manuscrito. Esta política persigue el doble objetivo anteriormente mencionado de ganar potenciales autores y lectores y, a la sazón, mejorar la calidad de la revista ${ }^{2}$. Esta calidad se mide, entre otros parámetros, por el número de citas que reciben sus artículos, del cual depende el factor de impacto (FI) de la revista. Esta edición bilingüe es usada sólo por 5 las revistas biomédicas españolas, pero se desconocen los resultados de esta estrategia. Éste fue el objetivo planteado en este trabajo.

\section{MATERIAL Y MÉTODOS}

El estudio se realizó utilizando el portal de Web of Knowledge (WoK) mediante el acceso gratuito facilitado por la Fundación Española de Ciencia y Tecnología (FECYT) (http://www.accesowok.fecyt.es/). El acceso se realizó el 12/11/2012. Las revistas estudiadas fueron las 5 del ámbito de la biomedicina clínica editadas en España, indexadas por SCI-Expanded y que Journal Citation Reports (JCR) categoriza, en el epígrafe de la lengua, como multilingües: Anales de Pediatría, Archivos de Bronconeumología, Medicina Clínica, Medicina Intensiva, y Nefrología. Los artículos incluidos fueron todos los publicados entre 20082012, excepto para Anales de Pediatría que sólo dispone de artículos indexados entre 2009-2012. Se contabilizaron los artículos originales ("Articles" en SCI-Expanded), los artículos sin autores españoles, las citas recibidas, los artículos citantes sin autor español, el idioma y las nacionalidades de los autores de estos artículos y de los artículos citantes.

Las variables cualitativas se expresan en valores absolutos y porcentajes, y las cuantitativas en medias, medianas y rangos intercuartílicos (RIC). La comparación entre los resultados obtenidos por los artículos publicados en español y en inglés se realizó mediante el test de la ji al cuadrado y el test no paramétrico de la U de MannWhitney. Las diferencias se consideraron estadísticamente si $\mathrm{p}<0,05$. Por el carácter público de los datos, no fue precisa la aprobación por un Comité de Ética de Investigación Clínica.

\section{RESULTADOS}

Se incluyeron un total de 4.296 documentos, de los que 85 (2,0\%) fueron publicados en inglés. Hubo diferencias significativas entre las características de los artículos publicados en inglés y español (Tabla 1): los primeros incluyeron porcentajes superiores de originales y sin autoría española, tuvieron más citas y fueron citados por más artículos. Además, el porcentaje de artículos citantes sin autoría española fue también superior para los artículos en inglés. 
Tabla 1. Principales características de los artículos publicados entre enero de 2008 y octubre de 2012 en las 5 revistas analizadas.

\begin{tabular}{|c|c|c|c|c|}
\hline & $\begin{array}{l}\text { Artículos totales } \\
\text { analizados } \\
(\mathrm{N}=4.269)\end{array}$ & $\begin{array}{l}\text { Artículos publicados } \\
\text { en español } \\
(\mathrm{N}=4.184)\end{array}$ & $\begin{array}{c}\text { Artículos publicados } \\
\text { en inglés } \\
(\mathrm{N}=85)\end{array}$ & $\mathbf{p}$ \\
\hline Artículos originales de investigación & 1.733 & 1.685 & 48 & \\
\hline $\begin{array}{l}\text { - Porcentaje de originales } \\
\text { respecto a artículos totales }\end{array}$ & 40,6 & 40,3 & 56,5 & 0,004 \\
\hline Artículos sin autor español & 370 & 333 & 37 & \\
\hline $\begin{array}{l}\text { - Porcentaje artículos sin autor } \\
\text { español respecto a artículos } \\
\text { totales }\end{array}$ & 8,7 & 8,0 & 43,5 & $<0,001$ \\
\hline Citas totales registradas & 4.543 & 4.371 & 172 & \\
\hline $\begin{array}{l}\text { - Citas por artículo publicado } \\
\text { (media / mediana / RIC) }\end{array}$ & $1,07 / 0 / 1$ & $1,01 / 0 / 1$ & $1,98 / 1 / 3$ & 0,006 \\
\hline $\begin{array}{l}\text { Numero artículos citantes } \\
\text { (total / sin autor español) }\end{array}$ & $3.557 / 1.248$ & $3.385 / 1.161$ & $172 / 87$ & \\
\hline $\begin{array}{l}\text { - Porcentaje artículos citantes } \\
\text { sin autor español respecto a } \\
\text { artículos citantes }\end{array}$ & 35,1 & 34,3 & 50,6 & $<0,001$ \\
\hline
\end{tabular}

\section{DISCUSIÓN}

La proporción de artículos publicados en inglés por las revistas españolas multilingües indexadas de SCI-Expanded es bastante marginal, pues supone sólo el $2 \%$. Esto es así a pesar que las normativas de estas revistas que se editan en forma bilingüe no contemplan específicamente una limitación del número de trabajos que pueden aceptar en inglés. Estos artículos publicados en inglés con mayor frecuencia son originales y de autores no españoles, y reciben más citas que los publicados en español, las cuales son realizadas, también con mayor frecuencia, por autores no españoles.

En los países de habla no inglesa, muchos investigadores deben escoger entre dirigir sus trabajos a revistas nacionales si pretenden una difusión local o hacia revistas en inglés si lo que persiguen es la máxima difusión o prestigio internacional ${ }^{3}$. Esto se debe en parte al déficit cuantitativo y cualitativo de revistas editadas en su propia lengua. Tal es el caso de España, que sólo tiene 78 revistas científicas incluidas en el JCR de 2011 y sólo 15 de ellas están posicionadas en el primer o segundo cuartil de sus respectivas especialidades ${ }^{4}$. La edición multilingüe de una revista podría paliar este defecto, pues permite que un autor que escribe su artículo en inglés consiga a la vez la difusión local de sus resultados y una mayor proyección internacional. Tal vez por ello, más de la mitad $(56,5 \%)$ de artículos publicados en inglés revisados en este artículo estaban firmados por autores españoles. Alternativamente, ello también podría justificarse por ser artículos posiblemente enviados originariamente a revistas de lengua inglesa y que, tras ser rechazados, son reenviados a revistas españolas que permiten publicar en inglés, lo cual ahorra tiempo en traducción. Se ha descrito que el escribir en inglés un artículo previamente rechazado en otra revista y el enviarlo a una revista de menor FI o de ámbito nacional incrementa sus posibilidades de ser aceptado ${ }^{5}$. 
Este trabajo remarca la importancia del inglés en la difusión y, más específicamente, en el número de citas recibidas por los trabajos de investigación. Este fenómeno de dominancia del inglés y de las publicaciones anglosajonas en las principales bases de datos es un sesgo bien conocido en el FI de las revistas científicas ${ }^{6}$. Trabajos publicados recientes han hallado que, en la categoría de medicina interna y general, tanto el FI como su incremento a lo largo del tiempo se asocian más con la publicación en lengua inglesa que con el país de origen ${ }^{7}$. Sin embargo, la repercusión que sobre el FI puede tener una política de edición multilingüe se adivina que es escasa por el bajo número de artículos implicados según el presente análisis. Sin duda la política multilingüe supone un abordaje más económico que el traducir con posterioridad al inglés algunos o todos los artículos publicados en español $\mathrm{y}$ ofrecerlos en formato electrónico. Esto último es cada vez más frecuente y facilita la citación internacional de los artículos ${ }^{8}$. Sin embargo, no debe olvidarse que a pesar de vivir en la era de la globalización, debe defenderse la riqueza que ofrece la expresión en diferentes lenguas ${ }^{9}$. En este sentido, es llamativo y de apreciar cómo revistas norteamericanas de primer nivel recientemente han empezando a traducir, para la versión electrónica, parte de sus contenidos al español ${ }^{10}$.

\section{BIBLIOGRAFÍA}

1. Reuters T. Journal Citation Reports. (consultado el 10/12/2012) Accesible en: http://wokinfo.com/media/pdf/jcrwebfs.pdf 2010

2. VILLAR J. El inglés, idioma internacional en Medicina. Med Clin (Barc) 1988; 91: 23-24.

3. Miró O, Valcárcel De la Iglesia MA, Cremades Pallas RM, Burillo-Putze G, Julián Jiménez A, MARTín-SÁnchez FJ. Producción científica de los urgenciólogos españoles durante el quinquenio 2005-2009 y comparación con el quinquenio 2000-2004. Emergencias 2012; 24: 164-174.

4. Miró O. El nuevo factor de impacto de EMERGENCIAS. Emergencias 2012; 24: 262-264.

5. Vinther S, Rosenberg J. Fate of manuscripts rejected by a non-English-language general medical journal: a retrospective cohort study. BMJ Open 2011; 1: e000147.

6. SegLen PO. Why the impact factor of journals should not be used for evaluating research. BMJ 1997; 314: 498-502.

7. Mueller PS, Murali NS, Cha SS, Erwin PJ, GHosh AK. The association between impact factors and language of general internal medicine journals. Swiss Med Weekly 2006; 136: 441-443.

8. Burillo-Putze G, Miró O. Emergencias en la red. Emergencias 2011; 23: 81-82.

9. Gregoire G, Derderian F, Le Lorier J. Selecting the language of the publications included in a meta-analysis: is there a Tower of Babel bias? J Clin Epidemiol 1995; 48: 159-163.

10. Miró O, Martín-SÁnchez FJ, Cone DC. La lengua española en la Medicina de Urgencias y Emergencias norteamericana. Emergencias 2012; 24 : 341-342. 